\title{
Bayesian Cramer-Rao Bound for Mobile Terminal Tracking in Mixed LOS/NLOS Environments
}

\author{
Carsten Fritsche, Anja Klein and Fredrik Gustafsson
}

\author{
Linköping University Post Print
}

\section{Tweet}

N.B.: When citing this work, cite the original article.

Carsten Fritsche, Anja Klein and Fredrik Gustafsson, Bayesian Cramer-Rao Bound for Mobile Terminal Tracking in Mixed LOS/NLOS Environments, 2013, IEEE Wireless Communications Letters, (2), 3, 335-338.

http://dx.doi.org/10.1109/WCL.2013.032013.130073

C2013 IEEE. Personal use of this material is permitted. However, permission to reprint/republish this material for advertising or promotional purposes or for creating new collective works for resale or redistribution to servers or lists, or to reuse any copyrighted component of this work in other works must be obtained from the IEEE.

http://ieeexplore.ieee.org/

Postprint available at: Linköping University Electronic Press

http://urn.kb.se/resolve?urn=urn:nbn:se:liu:diva-121382 


\title{
Bayesian Cramér-Rao Bound for Mobile Terminal Tracking in Mixed LOS/NLOS Environments
}

\author{
Carsten Fritsche, Member, IEEE, Anja Klein, Member, IEEE, and Fredrik Gustafsson, Fellow, IEEE
}

\begin{abstract}
A computational algorithm is presented for the Bayesian Cramér-Rao lower bound (BCRB) in filtering applications with measurement noise from mixture distributions with jump Markov switching structure. Such mixture distributions are common for radio propagation in mixed line- and non-lineof-sight environments. The newly derived BCRB is tighter than earlier more general bounds proposed in literature, and thus gives a more realistic bound on actual estimation performance. The resulting BCRB can be used to compute a lower bound on root mean square error of position estimates in a large class of radio localization applications. We illustrate this on an archetypical tracking application using a nearly constant velocity model and time of arrival observations.
\end{abstract}

Index Terms-Bayesian Cramér-Rao bound, jump Markov system, location tracking, non-linear filtering.

\section{INTRODUCTION}

Wireless location systems offering reliable estimates of the mobile terminal (MT) position have become an important research field over the last few years [1]. In this letter, we are concerned with tracking an MT in cellular radio networks with known base station (BS) positions, where time of arrival (TOA) estimates between BS and MT are used to estimate the MT position. Especially in urban areas, so called non-lineof-sight propagation (NLOS) can severely affect the position estimates of tracking algorithms. NLOS propagation occurs due to obstacles such as buildings, trees or hills, and hinders the signals to arrive via the direct (or LOS) path at the MT/BS, thus, leading to biased TOA estimates.

One approach to deal with the different propagation conditions is to introduce a noise model for LOS propagation and a noise model for NLOS propagation, where the transition between the LOS and NLOS mode is modeled with a Markov chain. This type of model has been proposed e.g. for TOA measurements, received signal strength and angle of arrival measurements, see for instance [2]-[4]. The area of developing multiple model-based filtering algorithms to solve this type of problem has become relative mature, see for instance [3]-[6]. We are interested here in the development of tight lower bounds on the positioning performance, which has been addressed so far only by a few authors [5], [6].

In [5], a conditional Cramér-Rao bound (CRB) for MT tracking using TOA measurements has been computed, which is based on an a-priori known mode sequence, a single MT trajectory and assuming zero process noise. A general method

C. Fritsche is with IFEN GmbH, Alte Gruber Str. 6, 85586 Poing, Germany. A. Klein is with the Department of Electrical Engineering, Communications Engineering Lab, Technische Universität Darmstadt, Merckstr. 25, 64289 Darmstadt, Germany. F. Gustafsson is with the Department of Electrical Engineering, Division of Automatic Control, Linköping University, SE-581 83 Linköping, Sweden, e-mail: \{carsten,fredrik\}@isy.liu.se, a.klein@nt.tudarmstadt.de. to compute a Bayesian CRB (BCRB) for multiple model filtering with unknown mode sequence is presented in [7], which is hereinafter referred to as Enumer-BCRB. The idea is to first compute the BCRB conditioned on a sequence of modes and then to evaluate the corresponding unconditional bound by averaging the conditional BCRB over all possible mode sequences.

The Enumer-BCRB for the MT tracking problem has been proposed in [6], where some of the expectations involved in computing the bound are further approximated using a decentralized extended Kalman filter (EKF) and deterministic sampling schemes. However, the Enumer-BCRB is known to be overly optimistic, i.e. the bound is often not tight and thus cannot predict the filtering performance. Thus, it is of great importance to develop a lower bound that is tight.

Recently, another type of BCRB for jump Markov systems for mode-dependent process models has been proposed which was shown to be sometimes tighter than the Enumer-BCRB [8]. In this letter, we modify the approach of [8] to the case of mode-dependent measurement models. The corresponding BCRB is derived for a system composed of a linear Gaussian process model and nonlinear measurement model with additive noise structure. It will be shown that for the particular case of MT tracking using a nearly constant velocity model and TOA measurements, the newly proposed BCRB is tighter than the Enumer-BCRB.

\section{SySTEM MOdEL}

Consider the following discrete-time jump Markov system, that is described by the following process and measurement equation

$$
\begin{aligned}
& \mathbf{x}_{k}=\mathbf{F}_{k} \mathbf{x}_{k-1}+\mathbf{v}_{k}, \\
& \mathbf{z}_{k}=\mathbf{h}_{k}\left(\mathbf{x}_{k}, r_{k}\right)+\mathbf{w}_{k}\left(r_{k}\right),
\end{aligned}
$$

where $\mathbf{z}_{k} \in \mathbb{R}^{n_{z}}$ is the measurement vector at discrete time $k$ and $\mathbf{x}_{k} \in \mathbb{R}^{n_{x}}$ is the state vector, $\mathbf{F}_{k}$ is an arbitrary linear mapping matrix and $\mathbf{h}_{k}$ is a non-linear mapping vector, both of appropriate size. The process and measurement noise vectors $\mathbf{v}_{k} \in \mathbb{R}^{n_{v}}$ and $\mathbf{w}_{k} \in \mathbb{R}^{n_{w}}$ are assumed to be mutually independent white processes. The process noise is distributed as $\mathbf{v}_{k} \sim \mathcal{N}\left(\mathbf{0}, \mathbf{Q}_{k}\right)$, where the matrix $\mathbf{Q}_{k}$ has to be invertible. The measurement noise can be distributed arbitrarily, but with known probability density function (pdf). The mode variable $r_{k}$ denotes a discrete-time Markov chain with $s$ states and transition probability matrix $\operatorname{Pr}\left\{r_{k} \mid r_{k-1}\right\}$. At time $k=0$, prior information about the state $\mathbf{x}_{0}$ and mode $r_{0}$ is available in terms of the pdf $p\left(\mathbf{x}_{0}\right)$ and probability mass function $\operatorname{Pr}\left\{r_{0}\right\}$. The initial state $\mathbf{x}_{0}$ is assumed to be Gaussian distributed with mean $\hat{\mathbf{x}}_{0}$ and covariance matrix $\mathbf{P}_{0}$.

In the following, let the operator $\mathbb{E}_{p(x)}\{\cdot\}$ denote expectation, 
where the subscript indicates the pdf that is used in the expectation operation. Furthermore, let $\mathbf{x}_{0: k}=\left[\mathbf{x}_{0}^{\top}, \ldots, \mathbf{x}_{k}^{\top}\right]^{\top}$ and $\mathbf{z}_{1: k}=\left[\mathbf{z}_{1}^{\top}, \ldots, \mathbf{z}_{k}^{\top}\right]^{\top}$ denote the collection of states and measurement vectors up to time $k$, and let $\hat{\mathbf{x}}_{0: k}\left(\mathbf{z}_{1: k}\right)$ denote any estimator of the sequence $\mathbf{x}_{0: k}$. The sequence of mode variables at time $k$ is denoted as $r_{1: k}^{i}=\left(r_{1}^{i}, r_{2}^{i}, \ldots, r_{k}^{i}\right)$, where $i=1, \ldots, s^{k}$. The gradient of a vector $\mathbf{u}$ is defined as $\nabla_{\mathbf{u}}=\left[\partial / \partial u_{1}, \ldots, \partial / \partial u_{n}\right]^{\top}$ and the Laplace operator is defined as $\Delta_{\mathbf{u}}^{\mathbf{t}}=\nabla_{\mathbf{u}}\left[\nabla_{\mathbf{t}}\right]^{\top}$.

\section{BAYESIAN CRAMÉR-RAO BOUND}

The BCRB for the sequence $\mathrm{x}_{0: k}$ provides a lower bound on the mean square error matrix for any estimator $\hat{\mathbf{x}}_{0: k}\left(\mathbf{z}_{1: k}\right)$, and is defined as the inverse of the Bayesian information matrix (BIM) $\mathbf{J}_{0: k}$,

$\mathbb{E}_{p\left(\mathbf{x}_{0: k}, \mathbf{z}_{1: k}\right)}\left\{\left[\hat{\mathbf{x}}_{0: k}\left(\mathbf{z}_{1: k}\right)-\mathbf{x}_{0: k}\right]\left[\hat{\mathbf{x}}_{0: k}\left(\mathbf{z}_{1: k}\right)-\mathbf{x}_{0: k}\right]^{\top}\right\} \geq \mathbf{J}_{0: k}^{-1}$.

Here, the matrix inequality $\mathbf{A} \geq \mathbf{B}$ means that the difference $\mathbf{A}-\mathbf{B}$ is a positive semidefinite matrix [9]. The BCRB for the current state $\mathbf{x}_{k}$ is of particular interest, since this gives a lower bound on the performance of nonlinear filtering. It has been shown in [10], that the BCRB for $\mathbf{x}_{k}$ is given by the $\left(n_{x} \times n_{x}\right)$ lower-right submatrix of $\left[\mathbf{J}_{0: k}\right]^{-1}$.

In the following, an algorithm that numerically evaluates $\mathbf{J}_{0: k}$ for system models given by (1) is presented, from which finally the BCRB for $\mathbf{x}_{k}$ can be obtained. The Bayesian information matrix $\mathbf{J}_{0: k}$ is given by

$$
\mathbf{J}_{0: k}=\mathbb{E}_{p\left(\mathbf{x}_{0: k}, \mathbf{z}_{1: k}\right)}\left\{-\Delta_{\mathbf{x}_{0: k}}^{\mathbf{x}_{0: k}} \log p\left(\mathbf{x}_{0: k}, \mathbf{z}_{1: k}\right)\right\} .
$$

This matrix is decomposed using Bayes' rule, yielding

$$
\mathbf{J}_{0: k}=\mathbf{J}_{\mathbf{x}_{0: k}}+\mathbf{J}_{\mathbf{z}_{1: k}},
$$

where $\mathbf{J}_{\mathbf{x}_{0: k}}$ denotes the BIM of the prior:

$$
\mathbf{J}_{\mathbf{x}_{0: k}}=\mathbb{E}_{p\left(\mathbf{x}_{0: k}\right)}\left\{-\Delta_{\mathbf{x}_{0: k}}^{\mathbf{x}_{0: k}} \log p\left(\mathbf{x}_{0: k}\right)\right\},
$$

and where $\mathbf{J}_{\mathbf{z}_{1: k}}$ denotes the BIM of the data:

$$
\mathbf{J}_{\mathbf{z}_{1: k}}=\mathbb{E}_{p\left(\mathbf{x}_{0: k}, \mathbf{z}_{1: k}\right)}\left\{-\Delta_{\mathbf{x}_{0: k}}^{\mathbf{x}_{0: k}} \log p\left(\mathbf{z}_{1: k} \mid \mathbf{x}_{0: k}\right)\right\} .
$$

In case of a linear Gaussian process model, cf. (1a), and assuming that the initial state $\mathbf{x}_{0}$ is Gaussian distributed according to $\mathbf{x}_{0} \sim \mathcal{N}\left(\hat{\mathbf{x}}_{0}, \mathbf{P}_{0}\right)$, the BIM of the prior can be computed analytically from the relationship

$$
\begin{aligned}
& \mathbf{J}_{\mathbf{x}_{0: k}}= \mathbb{E}_{p\left(\mathbf{x}_{0: k}\right)}\left\{-\Delta_{\mathbf{x}_{0: k}}^{\mathbf{x}_{0: k}} \log p\left(\mathbf{x}_{0}\right)\right\} \\
& \quad+\sum_{n=1}^{k} \mathbb{E}_{p\left(\mathbf{x}_{0: k}\right)}\left\{-\Delta_{\mathbf{x}_{0: k}}^{\mathbf{x}_{0: k}} \log p\left(\mathbf{x}_{n} \mid \mathbf{x}_{n-1}\right)\right\} \\
&= \delta_{k+1}(1,1) \otimes \mathbf{J}_{\mathbf{x}_{0}}+\sum_{n=1}^{k} \delta_{k+1}(n, n) \otimes \mathbf{F}_{n}^{\top} \mathbf{Q}_{n}^{-1} \mathbf{F}_{n} \\
&-\delta_{k+1}(n, n+1) \otimes \mathbf{F}_{n}^{\top} \mathbf{Q}_{n}^{-1}-\delta_{k+1}(n+1, n) \otimes \mathbf{Q}_{n}^{-1} \mathbf{F}_{n} \\
&+\delta_{k+1}(n+1, n+1) \otimes \mathbf{Q}_{n}^{-1},
\end{aligned}
$$

where $\mathbf{J}_{\mathbf{x}_{0}}=\left[\mathbf{P}_{0}\right]^{-1}, \otimes$ denotes the Kronecker product and $\delta_{k+1}(i, j)$ denotes a $(k+1) \times(k+1)$ dimensional matrix whose elements are all zero except at the $i$-th row and the $j$-th column which is one. The expression in (7) describes a block tridiagonal matrix of growing dimension, whose elements were derived in [10]. The evaluation of the BIM of the data $\mathbf{J}_{\mathbf{z}_{1: k}}$ involves the computation of expectations, which are difficult to express analytically for measurement models of the form (1b). In the following, we focus on the numerical approximation of $\mathbf{J}_{\mathbf{z}_{1: k}}$. The expectation given in (6) can be reformulated as

$\mathbf{J}_{\mathbf{z}_{1: k}}=\mathbb{E}_{p\left(\mathbf{x}_{0: k}, \mathbf{z}_{1: k}\right)}\left\{\frac{\nabla_{\mathbf{x}_{0: k}} p\left(\mathbf{z}_{1: k} \mid \mathbf{x}_{0: k}\right)\left[\nabla_{\mathbf{x}_{0: k}} p\left(\mathbf{z}_{1: k} \mid \mathbf{x}_{0: k}\right)\right]^{\top}}{\left[p\left(\mathbf{z}_{1: k} \mid \mathbf{x}_{0: k}\right)\right]^{2}}\right\}$,

which can be numerically approximated according to

$$
\mathbf{J}_{\mathbf{z}_{1: k}} \approx \frac{1}{N} \sum_{j=1}^{N} \frac{\nabla_{\mathbf{x}_{0: k}} p\left(\mathbf{z}_{1: k}^{(j)} \mid \mathbf{x}_{0: k}^{(j)}\right)\left[\nabla_{\mathbf{x}_{0: k}} p\left(\mathbf{z}_{1: k}^{(j)} \mid \mathbf{x}_{0: k}^{(j)}\right)\right]^{\top}}{\left[p\left(\mathbf{z}_{1: k}^{(j)} \mid \mathbf{x}_{0: k}^{(j)}\right)\right]^{2}},
$$

where $\mathbf{x}_{0: k}^{(j)}$ and $\mathbf{z}_{1: k}^{(j)}, j=1, \ldots, N$ are independent and identically distributed vectors such that $\left(\mathbf{x}_{0: k}, \mathbf{z}_{1: k}\right) \sim p\left(\mathbf{x}_{0: k}, \mathbf{z}_{1: k}\right)$. In order to approximate $\mathbf{J}_{\mathbf{z}_{1: k}}$ as in (9), the quantities $p\left(\mathbf{z}_{1: k} \mid \mathbf{x}_{0: k}\right)$ and $\nabla_{x_{0: k}} p\left(\mathbf{z}_{1: k} \mid \mathbf{x}_{0: k}\right)$ have to be evaluated. A recursive method for computing these quantities, and thus $\mathbf{J}_{\mathbf{z}_{1: k}}$, is given in Algorithm 1. Note, that samples from $p\left(\mathbf{z}_{k} \mid \mathbf{x}_{k}^{(j)}, r_{k}^{(j)}\right)$ can be obtained by generating a realization $\mathbf{w}_{k} \sim p_{\mathbf{w}\left(r_{k}\right)}$ from the density that is associated to the $r_{k}^{(j)}$. This realization together with the given $\mathbf{x}_{k}^{(j)}$ is plugged into the measurement model (1b), in order to deliver a realization $\mathbf{z}_{k}^{(j)}$.

The computation of $\mathbf{J}_{\mathbf{z}_{1: k}}$ does not require an explicit summation over all possible mode sequences $r_{1: k}^{i}$, yielding a complexity which is in the order of $\mathcal{O}(N \cdot m \cdot k)$. However, for the computation of the BCRB for $\mathbf{x}_{k}$, the matrix inverse $\left[\mathbf{J}_{0: k}\right]^{-1}$ is required, whose complexity increases with time $k$ and which is in the order of $\mathcal{O}\left(\left[(k+1) n_{x}\right]^{3}\right)$. Thus, the algorithm becomes eventually impractical for state sequences of arbitrary length. In [10], the block tridiagonal property was used to find a recursive algorithm for computing the $\left(n_{x} \times n_{x}\right)$ lower-right submatrix of $\left[\mathbf{J}_{0: k}\right]^{-1}$. The presented algorithm, however, does not provide a matrix $\mathbf{J}_{\mathbf{z}_{1: k}}$ with such a property. In order to obtain a recursive algorithm with reduced computational complexity, ideas similar to those presented in [8] can be adopted, where certain matrix elements on the off-diagonal of $\mathbf{J}_{\mathbf{z}_{1: k}}$ are intentionally set to zero to preserve a block tridiagonal structure, thus, allowing a recursive computation of a submatrix of $\mathbf{J}_{0: k}$ from which the BCRB for $\mathbf{x}_{k}$ can be finally extracted.

\section{Mobile Terminal Tracking Example}

The MT state vector $\mathbf{x}_{k} \in \mathbb{R}^{n_{x}}$ to be estimated is composed of the two-dimensional position and velocity, i.e. $\mathbf{x}_{k}=\left[x_{k}, y_{k}, \dot{x}_{k}, \dot{y}_{k}\right]^{\top}$. The MT's movement is modeled with a nearly constant velocity model

$$
\mathbf{x}_{k}=\mathbf{F} \mathbf{x}_{k-1}+\mathbf{v}_{k-1},
$$

where

$$
\mathbf{F}=\mathbf{I}_{2} \otimes\left[\begin{array}{ll}
1 & T \\
0 & 1
\end{array}\right]
$$

$T$ is the sampling time and $\mathbf{I}_{2}$ is the identity matrix of size 2 . The process noise vector $\mathbf{v}_{k-1} \in \mathbb{R}^{4}$ is assumed to be zeromean Gaussian distributed with block-diagonal covariance matrix $\mathbf{Q}=\operatorname{diag}_{\mathrm{b}}([\boldsymbol{\Sigma}, \boldsymbol{\Sigma}])$, where

$$
\boldsymbol{\Sigma}=q\left[\begin{array}{cc}
T^{3} / 3 & T^{2} / 2 \\
T^{2} / 2 & T
\end{array}\right]
$$


$\overline{\text { Algorithm } 1 \text { Computation of Bayesian information matrix of }}$ the data $\mathbf{J}_{\mathbf{z}_{1: k}}$

(1) At time $k=0$, generate $\mathbf{x}_{0}^{(j)} \sim p\left(\mathbf{x}_{0}\right)$ and $r_{0}^{(j)} \sim \operatorname{Pr}\left\{r_{0}\right\}$ for $j=1, \ldots, N$, and define $\mathbf{x}_{0: 0}^{(j)}=\mathbf{x}_{0}^{(j)}$.

(2) For $k=1,2, \ldots$, and $j=1, \ldots, N$ do:

- Generate $\mathbf{x}_{k}^{(j)} \sim p\left(\mathbf{x}_{k} \mid \mathbf{x}_{k-1}^{(j)}\right)$ and set $\mathbf{x}_{0: k}^{(j)}=$ $\left[\mathbf{x}_{0: k-1}^{(j)}, \mathbf{x}_{k}^{(j)}\right]$. Generate $r_{k}^{(j)} \sim \operatorname{Pr}\left\{r_{k} \mid r_{k-1}^{(j)}\right\}, \mathbf{z}_{k}^{(j)} \sim$ $p\left(\mathbf{z}_{k} \mid \mathbf{x}_{k}^{(j)}, r_{k}^{(j)}\right)$ and set $\mathbf{z}_{1: k}^{(j)}=\left[\mathbf{z}_{1: k-1}^{(j)}, \mathbf{z}_{k}^{(j)}\right]$.

- Update the stored quantities $\operatorname{Pr}\left\{r_{k-1}\right\}, \quad p\left(\mathbf{z}_{1: k-1}^{(j)} \mid \mathbf{x}_{0: k-1}^{(j)}, r_{k-1}\right) \quad$ and $\nabla_{\mathbf{x}_{0: k-1}} p\left(\mathbf{z}_{1: k-1}^{(j)} \mid \mathbf{x}_{0: k-1}^{(j)}, r_{k-1}\right)$ using the relations:

$\operatorname{Pr}\left\{r_{k}\right\}=\sum_{r_{k-1}} \operatorname{Pr}\left\{r_{k} \mid r_{k-1}\right\} \operatorname{Pr}\left\{r_{k-1}\right\}$,

$p\left(\mathbf{z}_{1: k}^{(j)} \mid \mathbf{x}_{0: k}^{(j)}, r_{k}\right)=p\left(\mathbf{z}_{k}^{(j)} \mid \mathbf{x}_{k}^{(j)}, r_{k}\right) \sum_{r_{k-1}} \operatorname{Pr}\left\{r_{k-1} \mid r_{k}\right\}$ $\times p\left(\mathbf{z}_{1: k-1}^{(j)} \mid \mathbf{x}_{0: k-1}^{(j)}, r_{k-1}\right)$,

$\nabla_{\mathbf{x}_{0: k}} p\left(\mathbf{z}_{1: k}^{(j)} \mid \mathbf{x}_{0: k}^{(j)}, r_{k}\right)=\sum_{r_{k-1}} \operatorname{Pr}\left\{r_{k-1} \mid r_{k}\right\}\left[\left[\nabla_{\mathbf{x}_{0: k}} p\left(\mathbf{z}_{k}^{(j)} \mid \mathbf{x}_{k}^{(j)}, r_{k}\right)\right]\right.$ $\times p\left(\mathbf{z}_{1: k-1}^{(j)} \mid \mathbf{x}_{0: k-1}^{(j)}, r_{k-1}\right)+p\left(\mathbf{z}_{k}^{(j)} \mid \mathbf{x}_{k}^{(j)}, r_{k}\right)$

$\left.\times\left[\nabla_{\mathbf{x}_{0: k}} p\left(\mathbf{z}_{1: k-1}^{(j)} \mid \mathbf{x}_{0: k-1}^{(j)}, r_{k-1}\right)\right]\right]$.

where

$$
\operatorname{Pr}\left\{r_{k-1} \mid r_{k}\right\}=\frac{\operatorname{Pr}\left\{r_{k} \mid r_{k-1}\right\} \cdot \operatorname{Pr}\left\{r_{k-1}\right\}}{\operatorname{Pr}\left\{r_{k}\right\}} .
$$

- Evaluate $p\left(\mathbf{z}_{1: k}^{(j)} \mid \mathbf{x}_{0: k}^{(j)}\right)$ and $\nabla_{\mathbf{x}_{0: k}} p\left(\mathbf{z}_{1: k}^{(j)} \mid \mathbf{x}_{0: k}^{(j)}\right)$ as follows:

$$
\begin{aligned}
p\left(\mathbf{z}_{1: k}^{(i)} \mid \mathbf{x}_{0: k}^{(j)}\right) & =\sum_{r_{k}} p\left(\mathbf{z}_{1: k}^{(j)} \mid \mathbf{x}_{0: k}^{(j)}, r_{k}\right) \operatorname{Pr}\left\{r_{k}\right\}, \\
\nabla_{\mathbf{x}_{0: k}} p\left(\mathbf{z}_{1: k}^{(j)} \mid \mathbf{x}_{0: k}^{(j)}\right) & =\sum_{r_{k}}\left[\nabla_{\mathbf{x}_{0: k}} p\left(\mathbf{z}_{1: k}^{(j)} \mid \mathbf{x}_{0: k}^{(j)}, r_{k}\right)\right] \operatorname{Pr}\left\{r_{k}\right\} .
\end{aligned}
$$

- Evaluate the Bayesian information matrix of the data $\mathbf{J}_{\mathbf{z}_{1: k}}$ according to (9).

and $q$ represents the process noise intensity level. It is further assumed that the MT is measuring the time a radio signal requires to propagate from the $m$-th $\mathrm{BS}$ to the $\mathrm{MT}$, where $m=1, \ldots, n_{z}$. In order to simplify the analysis, time synchronization among all BSs and the MT is assumed. The resulting TOA measurements are multiplied by the speed of light, yielding distance estimates, that are collected in the vector $\mathbf{z}_{k} \in \mathbb{R}^{n_{z}}$.

The switching between LOS and NLOS propagation conditions is modeled for each TOA with a 2-state Markov chain, where $r_{k}^{(m)}=1$ is assigned to the event "LOS" and $r_{k}^{(m)}=2$ is assigned to the event "NLOS". The $n_{z}$ Markov chains are combined into a single, augmented Markov chain, described by the mode variable $r_{k}$, that is assumed to be among the $s=2^{n_{z}}$ possible modes $r_{k} \in\left\{1, \ldots, 2^{n_{z}}\right\}$. As long as the TOA measurements are collected from BSs located at different sites, the LOS/NLOS transitions among different measurements can be assumed to be independent. In this case, the transition probability matrix $\Pi$ of the augmented Markov chain can be expressed in terms of the transition probability matrices $\Pi_{m}$ of the individual measurements according to $\boldsymbol{\Pi}=\boldsymbol{\Pi}_{1} \otimes \boldsymbol{\Pi}_{2} \otimes \cdots \otimes \boldsymbol{\Pi}_{n_{z}}$.

The effects of different propagation conditions can be taken into account by introducing a mode-dependent measurement noise vector, so that the model for the TOA measurements can be written as

$$
\mathbf{z}_{k}=\mathbf{h}_{k}\left(\mathbf{x}_{k}\right)+\mathbf{w}_{k}\left(r_{k}\right)
$$

where $\mathbf{h}_{k}\left(\mathbf{x}_{k}\right)=\left[h_{k}^{(1)}\left(\mathbf{x}_{k}\right), \ldots, h_{k}^{\left(n_{z}\right)}\left(\mathbf{x}_{k}\right)\right]^{\top}$, and $h_{k}^{(m)}\left(\mathbf{x}_{k}\right)$ is the Euclidean distance between the MT and the $m$-th BS. In LOS propagation conditions, each TOA measurement is only corrupted by system noise, which is described by a zeromean Gaussian distribution with variance $\sigma_{\text {LOS }}^{(m), 2}$. In NLOS conditions, two additive and independent error sources occur, namely system noise and errors resulting from NLOS propagation. In the present analysis, the NLOS error is assumed to be Gaussian distributed with positive mean $\mu_{\mathrm{NLOS}}^{(m)}$ and variance $\sigma_{\mathrm{NLOS}}^{(m), 2}$ [3], [5], [6]. In this case, $\mathbf{w}_{k}\left(r_{k}\right)$ is Gaussian distributed with mean vector $\boldsymbol{\mu}\left(r_{k}\right)$ and diagonal covariance matrix $\mathbf{R}\left(r_{k}\right)$, whose elements are given by

$$
\begin{aligned}
& \mu\left(r_{k}^{(m)}\right)=\left\{\begin{array}{ccc}
0 & \text { for } & r_{k}^{(m)}=1 \\
\mu_{\mathrm{NLOS}}^{(m)} & \text { for } & r_{k}^{(m)}=2,
\end{array}\right. \\
& \sigma^{2}\left(r_{k}^{(m)}\right)=\left\{\begin{array}{cll}
\sigma_{\mathrm{LOS}}^{(m), 2} & \text { for } & r_{k}^{(m)}=1 \\
\sigma_{\mathrm{LOS}}^{(m), 2}+\sigma_{\mathrm{NLOS}}^{(m), 2} & \text { for } & r_{k}^{(m)}=2
\end{array}\right.
\end{aligned}
$$

respectively. For the problem at hand, the densities that are required to evaluate Algorithm 1 are given by $p\left(\mathbf{x}_{k} \mid \mathbf{x}_{k-1}\right)=$ $\mathcal{N}\left(\mathbf{x}_{k} ; \mathbf{F} \mathbf{x}_{k-1}, \mathbf{Q}\right)$ and $p\left(\mathbf{z}_{k} \mid \mathbf{x}_{k}, r_{k}\right)=\mathcal{N}\left(\mathbf{z}_{k} ; \mathbf{h}\left(\mathbf{x}_{k}\right)+\right.$ $\left.\boldsymbol{\mu}_{k}\left(r_{k}\right), \mathbf{R}_{k}\left(r_{k}\right)\right)$. The elements of the gradient vector $\nabla_{\mathbf{x}_{0: k}} p\left(\mathbf{z}_{k} \mid \mathbf{x}_{k}, r_{k}\right)$ can be determined from

$$
\begin{aligned}
& \nabla_{\mathbf{x}_{l}} p\left(\mathbf{z}_{k} \mid \mathbf{x}_{k}, r_{k}\right)= \\
& \left\{\begin{array}{cl}
p\left(\mathbf{z}_{k} \mid \mathbf{x}_{k}, r_{k}\right)\left[\nabla_{\mathbf{x}_{l}} \mathbf{h}_{k}^{\top}\left(\mathbf{x}_{k}\right)\right] \mathbf{R}_{k}^{-1}\left(r_{k}\right) & \text { for } l=k \\
\times\left[\mathbf{z}_{k}-\mathbf{h}\left(\mathbf{x}_{k}\right)-\boldsymbol{\mu}_{k}\left(r_{k}\right)\right] & \text { otherwise } \\
\mathbf{0} & \text { in }
\end{array}\right.
\end{aligned}
$$

where $l=1, \ldots, k$ holds.

\section{PERformance Evaluation}

The newly proposed BCRB is compared to the following bounds and filter performances: 1. The Enumer-BCRB using Monte Carlo methods, see [7] and references therein; 2. The KF-based interacting multiple model (IMM) distance smoother [3]; 3. The IMM-EKF [5].

Simulation Scenario: It is assumed that the MT receives $n_{z}=3$ TOA measurements from BSs located at $[-3 \mathrm{~km},-2 \mathrm{~km}],[3 \mathrm{~km}, 5 \mathrm{~km}]$ and $[6 \mathrm{~km}, 2 \mathrm{~km}]$. The MT trajectories are generated according to the model given in (10), with process noise intensity $q=0.5 \mathrm{~m}^{2} / \mathrm{s}^{3}$. The initial MT state vector $\mathbf{x}_{0}$ is Gaussian distributed with mean $\hat{\mathbf{x}}_{0}=[500 \mathrm{~m}, 500 \mathrm{~m}, 5 \mathrm{~m} / \mathrm{s}, 5 \mathrm{~m} / \mathrm{s}]^{\top}$ and covariance matrix $\mathbf{P}_{0}=\operatorname{diag}\left(\left[(50 \mathrm{~m})^{2},(50 \mathrm{~m})^{2},(2 \mathrm{~m} / \mathrm{s})^{2},(2 \mathrm{~m} / \mathrm{s})^{2}\right]\right)$. The sampling time is chosen as $T=0.2 \mathrm{~s}$ and the sample length is 100. The TOA measurements are generated using (11), with noise parameters set to $\sigma_{\mathrm{LOS}}^{(m)}=150 \mathrm{~m}, \sigma_{\mathrm{NLOS}}^{(m)}=409 \mathrm{~m}$ and $\mu_{\mathrm{NLOS}}^{(m)}=513 \mathrm{~m}$, where $m=1,2$ and 3 [3]. The LOS/NLOS 
transitions for each TOA measurement are modeled with a Markov chain, whose initial mode probabilities are set to $\operatorname{Pr}\left\{r_{0}^{(m)}=1\right\}=0.5$ and $\operatorname{Pr}\left\{r_{0}^{(m)}=2\right\}=0.5, \forall m$, and whose transition probabilities are given by $\operatorname{Pr}\left\{r_{k}^{(m)}=1 \mid r_{k-1}^{(m)}=1\right\}=$ 0.9 and $\operatorname{Pr}\left\{r_{k}^{(m)}=2 \mid r_{k-1}^{(m)}=2\right\}=0.9, \forall m$.

Simulation Results: In this section the simulation results are presented. All bounds and filters have been initialized with $\hat{\mathbf{x}}_{0}$ and $\mathbf{P}_{0}$ and the results are averaged over $N_{\text {MC }}=20000$ Monte Carlo runs. In Fig. 1, the root mean square error (RMSE) of the MT position is shown for the different filters and bounds. It can be observed that the newly proposed BCRB is tighter than the Enumer-BCRB. The IMM-KF algorithm has the worst performance, while the performance of the computationally more complex IMM-EKF is very close to the $\mathrm{BCRB}$. The fact that the BCRB is tighter than the Enumer$\mathrm{BCRB}$ is somehow expected, since the Enumer-BCRB is derived as an average bound over estimators that are conditioned on the mode sequence (i.e. the estimator "knows" the mode sequence $r_{1: k}$ ), while for the BCRB $r_{1: k}$ is explicitly treated as unknown. However, this relation does not hold always and depends on many factors such as the difference between the $s$ models (some are informative, i.e. small noise covariance $\mathbf{R}_{k}\left(r_{k}\right)$, whereas others are less informative, i.e. large noise covariance $\mathbf{R}_{k}\left(r_{k}\right)$ ), see also the discussion in [8], or the existence of a mean $\boldsymbol{\mu}_{k}\left(r_{k}\right)$. Recall that the computation of the BCRB depends on $\boldsymbol{\mu}_{k}\left(r_{k}\right)$, while the Enumer-BCRB does not.

Another example for the difference between the BCRB and the Enumer-BCRB is given in Table I, where the average MT position RMSE has been evaluated for different mode transition probability values. It is expected that with decreasing values for the transition probabilities, the performance of the different filtering algorithms should degrade, because the Markov chain becomes less informative. While the filtering algorithms and the BCRB follow this trend, the Enumer-BCRB behaves conversely. A possible explanation for this effect is the missing spread of the means term (between an estimator that "knows" and does not "know" the mode sequence) in the computation of the Enumer-BCRB [11, Lemma 2]. For large transition probability values, the average performance differences, and thus, the contribution of the spread of the means term, will be small, since the estimators that do not know the mode sequence are equipped with a highly informative Markov chain. For small transition probability values, the reverse is true and the Enumer-BCRB gives a relatively poor prediction of filter performance.

TABLE I

POSITION RMSE AVERAGED OVER TIME VS. TRANSITION PROBABILITIES

\begin{tabular}{c||c|c|c|c|c}
\hline \multicolumn{1}{c||}{} & \multicolumn{5}{c}{$\pi_{11}^{(m)}=\pi_{22}^{(m)}, \forall m$} \\
Method & 0.6 & 0.7 & 0.8 & 0.9 & 0.95 \\
\hline \hline IMM-KF & 61.6 & 61.1 & 60.6 & 59.7 & 59.4 \\
IMM-EKF & 54.6 & 54.4 & 53.9 & 52.2 & 51.5 \\
BCRB & 53.8 & 53.5 & 52.7 & 51.3 & 50.3 \\
Enumer-BCRB & 48.9 & 49.0 & 49.2 & 49.5 & 50.1 \\
\hline
\end{tabular}

\section{CONCLUSION}

We have investigated the problem of computing the BCRB for system models, where the measurement model exhibits a

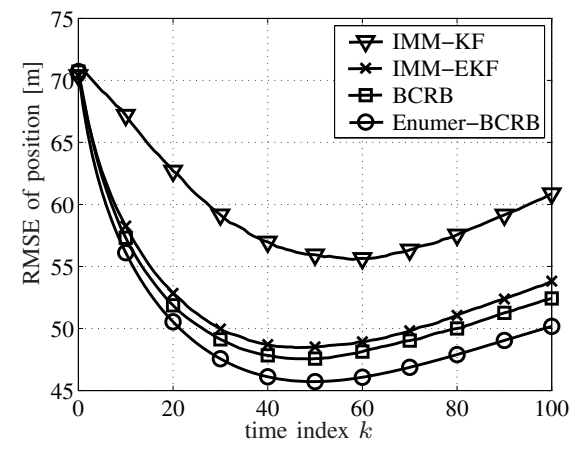

Fig. 1. Position RMSE vs. time step for the different filters and BCRBs based on $N=20000$ Monte Carlo runs.

Markovian switching structure. A novel algorithm for numerically evaluating the BIM of the complete state trajectory has been proposed, from which the BCRB is then extracted. The BIM can be divided into a sum of a prior BIM, which only depends on the process model, and a BIM of the data, which only depends on the measurement model. For the considered class of problems with a linear process model, the prior BIM becomes analytical. The non-linear observation model makes the BIM of the data intractable to compute analytically, and we propose to use Monte Carlo techniques to approximate this. For the problem of TOA-based MT tracking with linear dynamics in mixed LOS/NLOS environments, simulation results show that the newly proposed BCRB is tighter than a previously proposed Enumer-BCRB.

\section{REFERENCES}

[1] F. Gustafsson and F. Gunnarsson, "Mobile positioning using wireless networks: Possibilities and fundamental limitations based on available wireless network measurements," IEEE Signal Process. Mag., vol. 22, no. 4, pp. 41-53, Jul. 2005.

[2] K. Yu and Y. J. Gu, "Statistical NLOS identification based on AOA, TOA, and signal strength," IEEE Trans. Veh. Technol., vol. 58, no. 1, pp. 274-286, 2009.

[3] J.-F. Liao and B.-S. Chen, "Robust mobile location estimator with NLOS mitigation using IMM algorithm," IEEE Trans. Wireless Commun., vol. 5, no. 11, pp. 3002-3006, Nov. 2006.

[4] B.-S. Chen, C.-Y. Yang, F.-K. Liao, and J.-F. Liao, "Mobile location estimator in rough wireless environment using extended Kalman-based IMM and data fusion," IEEE Trans. Veh. Technol., vol. 58, no. 3, pp. 1157-1169, 2009.

[5] C. Fritsche, U. Hammes, A. Klein, and A. Zoubir, "Robust mobile terminal tracking in NLOS environments using interacting multiple model algorithm," in Proc. of IEEE International Conference on Acoustics, Speech and Signal Processing, Taipei, Taiwan, Apr. 2009, pp. 30493052.

[6] L. Chen, S. Ali-Löytty, R. Piché, and L. Wu, "Mobile tracking in mixed line-of-sight/non-line-of-sight conditions: Algorithm and theoretical lower bound," Wireless Personal Communcations, vol. 1, pp. 1-19, 2011.

[7] M. L. Hernandez, B. Ristic, and A. Farina, "A performance bound for manoeuvring target tracking using best-fitting Gaussian distributions," in Proc. of International Conference on Information Fusion, Philadelphia, PA, USA, Jul. 2005, pp. 1-8.

[8] L. Svensson, "On the Bayesian Cramér-Rao bound for Markovian switching systems," IEEE Trans. Signal Process., vol. 58, no. 9, pp. 4507-4516, Sept. 2010.

[9] H. L. van Trees, Detection, Estimation and Modulation Theory Part I. New York, NY, USA: John Wiley \& Sons, 1968.

[10] P. Tichavský, C. H. Muravchik, and A. Nehorai, "Posterior CramérRao bounds for discrete-time nonlinear filtering," IEEE Trans. Signal Process., vol. 46, no. 5, pp. 1386-1396, May 1998.

[11] B. Z. Bobrovsky, E. Mayer-Wolf, and M. Zakai, "Some classes of global Cramér-Rao bounds," The Annals of Statistics, vol. 15, no. 4, pp. 14211438, 1987. 